\title{
La integración entre cultura y economía. El caso de las Fiestas de Primavera de Sevilla
}

\author{
Luis Palma Martos a , María Luisa Palma Martos a , José Luis Martín \\ NAVARRO ${ }^{\text {a }}$ \\ a Universidad de Sevilla, Facultad de CC.EE., Avda. Ramón y Cajal, 1, 41018 Sevilla, España. E- \\ mail:1palma@us.es,mpalma@us.es,jomartin@us.es
}

\begin{abstract}
RESUMEN
Las Fiestas de Primavera de Sevilla (FPS) constituyen el principal evento cultural de la ciudad con importantes implicaciones sociales, turísticas y económicas. Nuestro trabajo analiza esta dimensión de la cultura de la ciudad en un doble plano. Por un lado, se lleva a cabo una caracterización de las FPS, prototipo de patrimonio cultural material e inmaterial. Por otro lado, se lleva a cabo un estudio de Impacto Económico, calculado a partir de la suma de tres efectos. En primer lugar, los efectos directos derivados del gasto necesario realizado por instituciones públicas y privadas para la celebración del evento. En segundo lugar, los efectos indirectos, producto del gasto efectuado por los participantes en los eventos. Por último, se estiman los efectos inducidos para la economía en su conjunto, mediante los multiplicadores calculados a partir de una tabla Input-Output. La principal contribución del artículo es abordar un estudio de impacto económico de un bien cultural complejo y de naturaleza compuesta, como ejemplo de valorización económica a través de la cultura. Los resultados muestran un impacto económico estimado para el año 2009 de 915,8 millones de euros, que representan un 4,66\% del PIB de la ciudad de Sevilla.
\end{abstract}

Palabras clave: Economía, Cultura, Bienes Culturales, Fiestas de Primavera de Sevilla, estudios de impacto.

\section{The Link between Culture and Economy. The Case of Spring Fiestas in Seville}

\begin{abstract}
Spring Fiestas in Seville (SFS) constitute the main cultural event that takes place in the city, with strong social, economic and touristic implications. Our paper analyzes this cultural dimension of the city from two perspectives. First, we analyze the SFS as a model of cultural heritage, both material and immaterial. Secondly, we make an economic impact study estimated as an addition of three effects: Direct effect that derivate from private and public institution's expenditures which are necessary in order to celebrate SFS; Indirect effect produced by expenditures of participants in the events and Induced effect estimated using multipliers derived from input-output tables. The main contribution of the paper is to analyze the economic impact of a complex cultural good of a mix nature, as an example of economic valuation through the culture. Results show an estimated economic impact for 2009 of 915.8 million of euros that means $4.66 \%$ of city of Seville GDP.
\end{abstract}

Keywords: Cultural Economics, Cultural Goods, Spring Fiestas in Seville, Impact Studies.

Clasificación JEL: Z11, R11, R15

Artículo recibido en octubre de 2013 y aceptado en diciembre de 2013

Artículo disponible en versión electrónica en la página www.revista-eea.net, ref. ə-32117 


\section{INTRODUCCIÓN}

Las Fiestas de Primavera de la ciudad de Sevilla constituyen un fenómeno cultural de fuerte arraigo popular. También suponen el evento cultural con implicaciones turísticas y económicas de mayor calado. En el ámbito social definen un perfil, característico de la ciudad, que las hacen indisociables del propio espíritu de la misma. Esto significa que no podría entenderse a Sevilla sin sus Fiestas de Primavera. Estas realidades han hecho que, desde diversas perspectivas, haya querido conocerse la naturaleza de estas manifestaciones y valorar su trascendencia en esferas tales como la histórica, antropológica, política, social, cultural y artística y, naturalmente, económica.

Nuestro trabajo explora esta dimensión de la cultura de la ciudad, desde una perspectiva económica, en un doble plano. Por un lado, mediante un esfuerzo caracterizador de las Fiestas de Primavera de Sevilla (FPS) como bien cultural; y en segundo lugar, ofreciendo datos económicos, obtenidos a partir de un análisis de impacto, que nos permitirán calibrar la importancia que, desde esta perspectiva, tienen estas manifestaciones.

Es nuestro objetivo general, presentar las FPS en ese doble plano, cultural y económico, que de un modo u otro, resultan indisociables si queremos entender su naturaleza. Más específicamente, nuestros esfuerzos han estado orientados a presentar, de forma integrada, las FPS como un evento enraizado en la ciudad, pero con una indudable trascendencia exterior; resulta en ese sentido un fuerte atractivo para el turismo cultural y de ocio. El otro objetivo específico se concreta en presentar la estimación del impacto económico de las FPS.

Hemos procedido a partir de dos aproximaciones metodológicas, propias de la Economía de la Cultura y como tal, urdidas desde una visión interdisciplinar. La caracterización de las FPS como un bien cultural supone un esfuerzo integrador para situar los eventos en los diferentes ámbitos de la Economía de la Cultura para lo que seguiremos el trabajo de Palma et al. (2013). En lo que respecta al estudio de Impacto, nos hemos situado en una posición canónica, adaptando los métodos tradicionales a la realidad objeto de estudio.

La principal contribución del trabajo estriba en ser el primero que ofrece el análisis del vínculo entre la economía y la cultura de las FPS, a partir de un estudio de impacto económico de las mismas consideradas como un todo. Los resultados en este sentido nos muestran la fuerte incidencia que estas manifestaciones, desarrolladas en el plazo de un mes, tienen en la vida de la ciudad. Si nos atenemos a los datos, un 4,66\% sobre el PIB, de impacto económico total, no es una cifra desdeñable.

El artículo se estructura, tras esta introducción en los siguientes apartados. En el apartado 2 se caracterizan las FPS desde los ámbitos que nos ofrece la Economía de la Cultura, resaltando la naturaleza de bien cultural de las mismas. 
En el apartado 3 se presenta una aproximación a la Economía de las FPS. Se esbozan los principales sectores y agentes involucrados, al objeto de trazar un marco en el que, en el último apartado, presentar los datos estimados a partir de un análisis de impacto. El trabajo termina con un apartado de Conclusiones y otro de Bibliografía.

\section{LA CARACTERIZACIÓN DE LAS FIESTAS DE PRIMAVERA DE SEVILLA COMO BIEN CULTURAL}

Las ferias, fiestas populares y festivales son eventos especiales con un fuerte componente cultural que juegan un papel significativo en la vida de una comunidad y en su desarrollo económico. Éstas han proliferado en número, popularidad y diversidad a lo largo de los últimos años (Gursoy et al., 2004, NEA 2010, Palma et al., 2013) y han sido cada vez más objeto de estudio dados los diversos beneficios que originan y que oscilan desde los puramente económicos (generación de renta y empleo), hasta su contribución a reforzar la identidad de una ciudad o región y a favorecer una mayor cohesión social (Chew 1998). La vinculación de estos eventos a un territorio específico los convierte en importantes atractivos turísticos que revitalizan, tanto la vida económica del mismo, como las tradiciones locales, impactando positivamente en los estándares de vida de la ciudad, además de favorecer la imagen de una ciudad o región.

Las ferias y fiestas pueden ser consideradas como eventos similares a un festival cultural, en la medida que son celebradas regularmente y evidencian un alto valor cultural y pueden considerase también un ejemplo de patrimonio cultural inmaterial (Del Barrio et al., 2012). Estas celebraciones no engloban un programa específico que comprenda una oferta cultural en sí mismo, que difiera de año a año, sino simplemente expresan una manifestación cultural y artística, basada en hábitos adquiridos que reflejan la seña de identidad de un grupo particular y que, debido a su naturaleza, son únicos, cualquiera que sea el lugar en el que se celebren. Desde este punto de vista, las fiestas y ferias culturales son un interesante campo de investigación en la medida en que se fusionan aspectos materiales e inmateriales y generan una fuerte atracción para el turismo no local. Tal vez uno de los mayores ejemplos en este sentido sean las Fiestas de Primavera de Sevilla (FPS) que están comprendidas por dos celebraciones o eventos: la Semana Santa, de carácter religioso, y la Feria de Abril, de carácter profano, y que constituyen el principal evento cultural de la ciudad cada año. Dichas Fiestas están fuertemente enraizadas en la tradición y son conocidas tanto a nivel nacional como internacional.

\subsection{Las Fiestas de Primavera de Sevilla}

Las Fiestas de Primavera de Sevilla pueden describirse como un bien cultural complejo que comprende dos elementos que las definen: su naturaleza com- 
puesta, al estar comprendidas por dos celebraciones de carácter distinto, y la amplia interacción social que se produce en ellas. Cada evento cultural es único y a pesar de tener características distintivas y diferenciadas comparten un denominador común: una alta proporción de valores culturales inmateriales. Ese denominador común nos va a permitir agruparlos bajo una misma denominación. Estas características son las siguientes:

1.-Ambos eventos se celebran de manera cíclica y regular. Tienen lugar durante la misma estación cada año, la Primavera ${ }^{1}$, de ahí su nombre, cuando el clima de la ciudad invita a la vida en la calle y el encuentro de los ciudadanos, lo que favorece una mayor interacción y cohesión social. Estas fiestas permiten la celebración de la vida que representa la Primavera y por tanto su triunfo frente al Invierno, la muerte. En este sentido entroncan con las fiestas del renacimiento de la naturaleza, de origen precristiano. También se caracterizan por una duración limitada y estable, dos semanas que se corresponden con la celebración de cada evento. Existe un fuerte componente de repetición en cada evento: los mismos horarios e itinerarios en el caso de la Semana Santa, y los mismos espacios para la Feria de Abril, lo cual dota a las fiestas de un carácter intemporal.

2. Estos eventos son expresiones culturales significativas de la vida social y turística de la ciudad lo cual implica un alto grado de participación y articulación social. Esta participación es favorecida por la existencia de vínculos institucionales que reflejan la adscripción de cada individuo a ciertos grupos sociales. Estos vínculos lo constituyen la pertenencia a las Hermandades o Cofradías $^{2}$ de la ciudad en Semana Santa, y ser socio o propietario de una caseta ${ }^{3}$ en la Feria de Abril. Estos vínculos actúan como factores clave de identidad cultural, reforzando la interacción social y la cohesión entre los ciudadanos.

Por tanto, dichas celebraciones generan una fuerte atracción no solo para los turistas de naturaleza cultural sino para los residentes en la ciudad. Éstos, de-

\footnotetext{
${ }^{1}$ El nombre de Fiestas de Primavera aparece a finales del siglo XIX y principios del Siglo XX cuando el Ayuntamiento de Sevilla empieza a editar carteles en los que se anuncia la celebración de las FPS englobando dos acontecimientos consecutivos: la Semana Santa, y la Feria de Abril. La más antigua es la Semana Santa que se remonta al siglo XVI, concretamente a 1521. Por su parte, la más moderna es la Feria de Abril, que nace en 1847 siendo su origen mercantil, ya que se trataba inicialmente de una feria de ganado semejante a las que se celebra-ban en otros lugares de España

2 En Sevilla existen actualmente 59 cofradías que procesionan durante la Semana Santa, distribuidas desde el Domingo de Ramos hasta el Domingo de Resurrección. Para un estudio más detenido sobre la tipología de Hermandades de Sevilla véase Moreno (1999, 2001).

${ }^{3}$ Según información proporcionada por La Delegación de Fiestas Mayores del Ayuntamiento de Sevilla para el año 2008, el número de casetas existentes era de 1047, 27 familiares de titularidad única, 499 familiares de titularidad compartida, 311 de diversas entidades privadas, 190 peñas, 1 Municipal, 6 de distritos municipales y 13 de servicios públicos.
} 
bido a la fuerte implicación de las mismas en la vida política, social y cultural de la ciudad, permanecen en ella para asistir a las Fiestas eligiendo, en muchos casos, la propia ciudad como opción de vacaciones.

\subsection{Las Fiestas de Primavera como bien cultural}

Como hemos expresado anteriormente, las FPS comparten un claro componente cultural, ya que son acontecimientos que transmiten mensajes simbólicos a quienes participan en ellas, entrañan un componente creativo, e incorporan, almacenan o generan formas de valor que no son expresables en su totalidad en términos monetarios ${ }^{4}$. Lo anterior nos permite hablar de las FPS de Sevilla como capital cultural de la ciudad (Throsby 1999). Aunque las FPS evidencian un capital cultural tangible acumulado (esculturas, edificios históricos), la característica distintiva de las FPS, si las comparamos con otros eventos culturales, es que representan un prototipo cultural de patrimonio intangible o inmaterial (música, literatura, tradiciones, valores), en la medida que se trata de una manifestación cultural que tiene fuertes raíces en la ciudad y que fusiona tradiciones que se han mantenido a lo largo del tiempo con nuevas formas de expresiones artísticas que reflejan la evolución de la sociedad y que también representan las señas de identidad de la ciudad, y la manera en que la cultura es entendida, creada, reproducida y consumida.

\section{Tabla 1}

Fiestas de Primavera de Sevilla.

Caracterización según los ámbitos de la economía de la cultura

\begin{tabular}{|c|c|c|}
\hline $\begin{array}{l}\text { ÁREAS DE } \\
\text { ECONOMÍA DE LA } \\
\text { CULTURA }\end{array}$ & SEMANA SANTA & FERIA DE ABRIL \\
\hline Artes representativas & $\begin{array}{l}\text { La ciudad como un gran escenario donde se representa } \\
\text { la pasión de Cristo a través de los pasos de las diver- } \\
\text { sas cofradías. Estilos diferentes de procesionar, serias } \\
\text { y de barrio. Los pasos van acompañados por música } \\
\text { que varía según las cofradías (marchas procesionales, } \\
\text { banda de cornetas y tambores, música de cámara) y } \\
\text { saetas. Forma diversa de componer los pasos de Cristo } \\
\text { y Virgen (distribución de las imágenes, ropajes, flores, } \\
\text { etc.).Forma de levantar los pasos y llevarlos. }\end{array}$ & $\begin{array}{l}\text { El baile, el toque, el cante en torno a una } \\
\text { música específica [sevillanas] y con } \\
\text { vestuario característico (trajes de fla- } \\
\text { menca, trajes de corto) dentro y fuera de } \\
\text { las casetas. Paseo de caballos. }\end{array}$ \\
\hline $\begin{array}{l}\text { Patrimonio material e } \\
\text { inmaterial }\end{array}$ & $\begin{array}{l}\text { Patrimonio material, lglesias, Catedral, edificios } \\
\text { emblemáticos: Giralda, Ayuntamiento. } \\
\text { Más el patrimonio inmaterial, tradiciones preservadas } \\
\text { por las Hermandades o Cofradías. }\end{array}$ & $\begin{array}{l}\text { Fundamentalmente patrimonio Inmaterial. }{ }^{*} \\
\text { Espacios y tiempos en la Feria de Abril. } \\
\text { Construcción efímera representativa de la } \\
\text { ciudad (portada de la Feria) }{ }^{\star \star}\end{array}$ \\
\hline
\end{tabular}

${ }^{4}$ Las FPS como bienes culturales generan beneficios externos tales como valor estético o espiritual, más en el caso de la Semana Santa que de la Feria, valor social, sirviendo como vínculo que reafirma el sentido de identidad asociado con la ciudad y su vida cultural, valor histórico, valor simbólico anteriormente mencionado, y finalmente valor de autenticidad. 
Tabla 1 (continuación)

Fiestas de Primavera de Sevilla.

Caracterización según los ámbitos de la economía de la cultura

\begin{tabular}{lll}
\hline $\begin{array}{c}\text { AREAS DE } \\
\text { ECONOMÍA DE LA } \\
\text { CULTURA }\end{array}$ & \multicolumn{1}{c}{ SEMANA SANTA } & \multicolumn{1}{c}{ FERIA DE ABRIL } \\
\hline & $\begin{array}{l}\text { Escultura (imaginería), Artesanía (orfebrería, cerería, } \\
\text { bordados, carpintería, dorado, etc.) Diseño del cartel de } \\
\text { la Semana Santa. }\end{array}$ & $\begin{array}{l}\text { Artesanía (trajes de flamenca, mantones, } \\
\text { zapatos, flores, etc.), trajes de corto, } \\
\text { sombreros, sajones, adornos de caballos, } \\
\text { carruajes, etc. Diseño del cartel de la } \\
\text { Feria y de la Portada. }\end{array}$ \\
\hline $\begin{array}{l}\text { Industrias creativas } \\
\text { tradicionales }\end{array}$ & $\begin{array}{l}\text { Libros, prensa, CDs música, retransmisión por radio y TV, DVDs, fotografía, reproducción de } \\
\text { carteles, cine. }\end{array}$ \\
\hline
\end{tabular}

${ }^{*}$ Nos basamos en el concepto de patrimonio inmaterial definido por la Unesco (2009) ${ }^{5}$ ** Candidata por la Unesco a ser declarada Patrimonio Inmaterial.

Fuente: Palma et al. (2013) "Determinants of cultural and popular celebration attendance: the case Study of Seville Spring Fiestas", Journal of Cultural Economics, vol.37 (1), p.91.

La Tabla 1 muestra las características comparadas de los dos eventos que conforman las FPS y que nos permiten definirlas como bienes culturales. Las FPS aúnan características asociadas a las distintas tipologías de bienes culturales (patrimonio material e inmaterial, artes visuales, artes representativas y distintas industrias creativas tradicionales), por lo que no se incluyen en un ámbito específico dentro de la amplia delimitación del sector cultural. Esto significa que la enorme complejidad que envuelve a los eventos hace su análisis extremadamente complicado en términos económicos y difícilmente comparables a otros bienes.

Ambos eventos muestran características vinculadas a las artes escénicas y tienen lugar en espacios públicos al aire libre. La Semana Santa se desarrolla en las calles de la ciudad, que se convierten en un gran escenario en el que la $\mathrm{Pa}$ sión de Cristo es representada por las Hermandades que realizan la carrera ofi$\mathrm{cial}^{6}$, situada en el centro histórico de la ciudad, donde se encuentran los principales monumentos tales como la Catedral, la Giralda, o el Ayuntamiento. En contraposición, la Feria de Abril tiene lugar en un espacio público específico acotado, situado en un extenso terreno ubicado en las lindes de la ciudad, concretamente en el barrio de Los Remedios, denominado El Real de la Feria. Este lugar, en el que se ubican las casetas, pretende ser una reproducción efímera de la ciudad misma, y donde los que participan en ella, cantan y bailan en diferen-

\footnotetext{
${ }^{5}$ Usos y representaciones, conocimientos y técnicas de las comunidades, tradiciones y expresiones orales, artes del espectáculo, música tradicional, danza. Usos sociales, rituales y actos festivos, técnicas artesanales tradicionales, y costumbres.

${ }^{6}$ Por Carrera Oficial se entiende el recorrido que realizan las diversas Hermandades de la ciudad desde la plaza de la Campana hasta la Catedral de Sevilla. Es el único recorrido común de todas las Hermandades que hacen estación de penitencia en la Semana Santa, ya que el resto del recorrido es diverso, dependiendo de la Iglesia de la que parta o tenga su sede cada Hermandad.
} 
tes estilos, tanto en el interior como en el exterior de las casetas, y en el caso de las mujeres frecuentemente ataviadas con el traje tradicional (traje de flamenca). Ambos eventos son una muestra del patrimonio material e inmaterial de la ciudad apoyándonos en dos argumentos (del Barrio et al., 2012): el primero se basa en que dichos eventos reflejan un bien de experiencia, de manera que los que asisten disfrutan de una representación en vivo única que expira en el momento que se produce (experiencia al contemplar el paso de una Hermandad o momento vivido en el interior o exterior de las casetas durante la Feria) y cuya permanencia en forma material depende en muchas ocasiones de la explotación por parte de la industria creativa tradicional. Dichos eventos dan lugar a diferentes bienes culturales: hay literatura relacionada, CDs sobre la música de Semana Santa (marchas procesionales y saetas), CDs de sevillanas, DVDs o cine específico de las Fiestas de Primavera, etc. El segundo es que tales eventos se apoyan en un capital cultural específico de la ciudad (imaginería y artesanías sevillanas, implicaciones en el diseño de moda española en el caso de la Feria, etc), y basado en tal bien, contemplado como un recurso, muestran y reinterpretan un legado cultural, de manera que contribuyen a reafirmar la identidad de los que participan en ellas.

Las FPS también evidencian ciertas características de bienes públicos, al ser bienes no rivales y no excluibles, en la medida que la admisión y participación es libre. Únicamente cuando se contempla la Semana Santa en la carrera oficial, para lo que es necesario pagar un precio por el alquiler de un palco o una silla, o cuando se visitan las casetas privadas en la Feria de Abril, en las que es necesario para acceder ser socio o tener invitación, las FPS tienen las propiedades de bienes club (Buchanan 1965).

\section{Características de la participación en las FPS}

La participación en las FPS hace referencia a un tipo de participación activa (O’Hagan, 1996). Ésta puede verse condicionada, por la existencia de un factor clave para el individuo como son los vínculos institucionales: ser miembro de una hermandad en la Semana Santa y el ser propietario o socio de una caseta en la Feria de Abril. En ambos casos implica no solo contemplar el evento como espectador sino también formar parte activa del mismo, incorporándose al cortejo de las cofradías en una de sus múltiples formas: nazareno, costalero, penitente, músico de banda, cuerpo de capataces o cantando saetas, en el caso de Semana Santa, o participando tanto en el cante como en el baile de las sevillanas, que se producen fundamentalmente en el interior de las casetas, en el caso de la Feria de Abril.

Aunque los vínculos que favorecen una mayor participación activa son el resultado de tradiciones familiares y son mantenidos de generación en generación, también pueden ser analizados como un proceso de selección ya que suponen un 
coste de mantenimiento en términos financieros. Estos vínculos contribuyen a crear ciertos gustos o preferencias por tales eventos que se forjan desde la niñez $\mathrm{y}$ que pueden favorecer una mayor intensidad en la asistencia a los mismos, lo que va a dar lugar a diferentes perfiles entre aquellos que asisten a las FPS.

En contraste con este tipo de participación, también puede darse un tipo de participación pasiva como simple espectador, que en el caso de la Semana Santa supone el contemplar el evento en la calle o incluso en la carrera oficial y en el caso de la Feria de abril implica, para aquellos que no son propietarios o socios de una caseta, realizar una visita panorámica y disfrutar del valor estético y de la atmósfera de la ciudad (portada de feria, colorido, paseo de caballos, música, baile, cante, etc.) pero cuyo acceso queda limitado a la entrada a las casetas de distritos o, por invitación, a las particulares.

\section{ECONOMÍA DE LAS FIESTAS DE PRIMAVERA DE SEVILLA. UN ANÁLISIS DE IMPACTO}

\subsection{La economía de las Fiestas de Primavera en Sevilla. Una aproximación}

Las Fiestas de Primavera en Sevilla tienen un indudable peso en la actividad económica de la ciudad que se extiende a lo largo de casi todo el año aunque tiene su punto álgido en torno a la celebración de ambas fiestas que tiene lugar en unas fechas variables pero siempre comprendidas entre mitad de marzo y primera semana de mayo.

Son dos los ámbitos que desde el punto de vista económico se pueden distinguir atendiendo la extensión temporal de la realización de las actividades económicas asociadas. Por un lado, la realización de actividades productivas y de servicios destinados a la tener disponibles los diferentes elementos en las fechas establecidas, si bien considerando la estacionalidad inherente a este tipo de actividades. A modo de ejemplo, el sector textil de fabricación de trajes de flamenca o túnicas de nazarenos, las artesanías asociadas a las actividades procesionales, como el bordado, el tallado o la orfebrería, etc. Otras actividades económicas se desarrollan de forma puntual a lo largo de un periodo de tiempo relativamente corto, en torno a la celebración de las fiestas. Entre este tipo de actividades económicas destacaríamos todas las actividades desarrolladas por el Ayuntamiento para el ordenado discurrir de las fiestas (montaje de infraestructuras efímeras, transporte público, limpieza, policía, etc.) o las actividades del sector privado asociadas por ejemplo al sector turístico (hostelería, alojamiento, transporte, etc.), montaje y explotación del parque de atracciones de la Feria.

Los sectores económicos más involucrados con la celebración de las fiestas de primavera en Sevilla son, el sector público, relacionado con todas las actividades económicas de prestación de servicios públicos; el sector industrial, con actividades del sector textil, artes gráficas, producción discográfica, artesanías 
asociadas (bordado, taller de escultura, tallado, carpintería, dorado, conservación de obras de arte), alimentación y bebidas; el sector de la construcción, sobre todo vinculado a la arquitectura efímera: casetas, portada de Feria, palcos y sillas en Semana Santa, y el sector servicios: transporte, turismo, hostelería, restauración, información y comunicación.

Al ser la actividad de un fuerte carácter estacional, la producción y el empleo asociados también tienen ese carácter, aunque con una actividad de mantenimiento el resto del año, ya que muchas de las industrias localizadas en Sevilla tienen un fuerte componente exportador a otras ciudades donde se celebran fiestas de características semejantes.

En el apartado de actividad económica asociada a estos eventos también se debe tener en cuenta que a lo largo del año se celebran en Sevilla encuentros empresariales de carácter nacional e internacional referidos a elementos característicos de las Fiestas de Primavera como el dedicado a los trajes de flamenca, Salón Internacional de la Moda Flamenca (SIMOF), que se celebra desde 1994, el Salón del Caballo de pura raza (SICAB), que se celebra desde 1991, o la Muestra Nacional de Artesanía Cofrade (MUNARCO) que se celebró entre 1997 y 2007. El Ayuntamiento de Sevilla planteó en 2005 la creación de un Parque Empresarial de Arte Sacro y Afines dedicado a la actividad, con un tamaño de hasta 100 talleres con el fin de localizar las actividades de este tipo de negocios que se desarrollan en lugares dispersos de la ciudad y área metropolitana.

En la primera década del siglo XXI empiezan a proliferar análisis de impacto económico, con el fin de justificar y poner en valor la celebración de eventos de carácter cultural tales como festivales, exposiciones, congresos, capitalidad cultural, o celebración de la Semana Santa, o Feria.

Desde el punto de vista económico, no han sido muchos los estudios que se han llevado a cabo sobre la materia de la que trata este trabajo. Los análisis de impacto económico de la Semana Santa de Sevilla comenzaron a hacerse a finales de los años 1990 cuando el Ayuntamiento y las organizaciones empresariales de la ciudad realizaron un trabajo en este sentido, este trabajo se amplió en 2009. Lazzeretti (2008 y 2011) ha realizado trabajos sobre distritos culturales tomando como ejemplos los casos de la Semana Santa y la Feria. Entre 2008 y 2010, Palma et al. (2008, 2010a, 2010b). Llevaron a cabo diversos estudios para la estimación del impacto económico de la celebración de las FPS. La propuesta metodológica inicial de Palma et al. (2008) ha servido de modelo para la realización del análisis de impacto económico referido a la Semana Santa de Guatemala (Grupo Satélite, 2013). En esos mismos años se han realizado propuestas similares para estimar el impacto económico de la celebración de la Semana Santa en diversas ciudades españolas como Córdoba (Analistas Económicos de Andalucía, 2009) o Palencia (Lafuente, Devesa y Sanz, 2012). En la Tabla 2 se 
ofrece un resumen de las características más relevantes de los diversos estudios relacionados.

Tabla 2

Trabajos de análisis de impacto de la Semana Santa

\begin{tabular}{|c|c|c|c|c|}
\hline TRABAJO & TÍTULO & OBJETIVO & METODOLOGÍA & RESULTADOS \\
\hline $\begin{array}{l}\text { Analistas } \\
\text { Económicos de } \\
\text { Andalucía (2009) }\end{array}$ & $\begin{array}{l}\text { Semana Santa. } \\
\text { Efectos } \\
\text { socioeconómicos de } \\
\text { la Semana Santa en } \\
\text { la ciudad de Córdoba }\end{array}$ & $\begin{array}{l}\text { Estimar impacto } \\
\text { económico de la } \\
\text { celebración de la S.S. en } \\
\text { Córdoba }\end{array}$ & $\begin{array}{l}\text { Metodología de impacto } \\
\text { económico con análisis de } \\
\text { efectos directos, indirectos e } \\
\text { inducidos. Realización de } \\
\text { encuestas a asistentes entre } \\
\text { los días } 5 \text { y } 12 \text { de abril de } \\
2009 \text {. Número de encuestas } \\
833\end{array}$ & $\begin{array}{l}\text { Impacto Económico } \\
\text { Total } 42.064 .210 .47 € \\
1.682 \text { puestos de } \\
\text { trabajo }\end{array}$ \\
\hline $\begin{array}{l}\text { Confederación } \\
\text { Empresarios de } \\
\text { Sevilla y Cámara } \\
\text { de Comercio de } \\
\text { Sevilla (2009) }\end{array}$ & $\begin{array}{l}\text { El impacto de la } \\
\text { celebración de la SS } \\
\text { Sevilla } 2008 . \\
\text {-Reelaboración y } \\
\text { actualización de un } \\
\text { trabajo anterior } \\
\text { realizado en 1999- }\end{array}$ & $\begin{array}{l}\text { Trabajo de campo en los } \\
\text { años } 2007 \text { y } 2008 \text { : } \\
\text { a) Entrevistas a } \\
\text { profesionales y } \\
\text { artesanos y entidades } \\
\text { turísticas de ciudad } \\
\text { b) Realización de } 2.088 \\
\text { encuestas, a asistentes } \\
\text { ( } 300 \text { encuestas diarias) } \\
\text { sobre gasto realizado }\end{array}$ & $\begin{array}{l}\text { Tres ámbitos: } \\
\text { 1. Artesanos y profesionales } \\
\text { 2. Flujos económicos } \\
\text { 3. Efecto turístico de la S.S. } \\
\text { y su repercusión } \\
\text { promocional de la ciudad }\end{array}$ & $\begin{array}{l}\text { Impacto económico } \\
\text { total estimado como } \\
\text { agregación de gastos } \\
\text { de diversos agentes } \\
\text { económicos, en torno } \\
\text { a } 160.000 .000 € \text {. } \\
\text { Hay que añadir el } \\
\text { impacto turístico y } \\
\text { promocional }\end{array}$ \\
\hline $\begin{array}{l}\text { Lazzeretti, L. } \\
\text { (2011) }\end{array}$ & $\begin{array}{l}\text { Culture as a source } \\
\text { for growth and } \\
\text { change: some } \\
\text { evidences from } \\
\text { cultural clusters in } \\
\text { Andalusia }\end{array}$ & $\begin{array}{l}\text { Análisis clusters culturales } \\
\text { en Andalucía. Especial } \\
\text { referencia a la Semana } \\
\text { Santa y Feria de Abril de } \\
\text { Sevilla. Y flamenco }\end{array}$ & $\begin{array}{l}\text { Revisión del factor cultural } \\
\text { como recurso estratégico en } \\
\text { el nuevo milenio }\end{array}$ & $\begin{array}{l}\text { Trabajo de tipo } \\
\text { cualitativo }\end{array}$ \\
\hline $\begin{array}{l}\text { Palma, et al. } \\
(2010 a, 2010 b)\end{array}$ & $\begin{array}{l}\text { Perfil de los } \\
\text { Asistentes e Impacto } \\
\text { Económico de la } \\
\text { Semana Santa y Feria } \\
\text { de Abril de Sevilla en } \\
\text { el año } 2009\end{array}$ & $\begin{array}{l}\text { Análisis del perfil de los } \\
\text { asistentes a la Semana } \\
\text { Santa y la Feria de abril de } \\
\text { Sevilla en el año } 2009 . \\
\text { Estimación del impacto } \\
\text { económico de las } \\
\text { celebraciones }\end{array}$ & $\begin{array}{l}\text { Metodología de encuestas } \\
310 \text { en Semana Santa y } 284 \\
\text { en Feria de abril } \\
\text { Aplicación de los modelos } \\
\text { de impacto a partir de la TIO } \\
\text { de Andalucía }\end{array}$ & $\begin{array}{l}\text { Semana Santa } \\
240.305 .044 € \\
\text { Feria Abril } \\
675.516 .437 €\end{array}$ \\
\hline $\begin{array}{l}\text { Lafuente, V., } \\
\text { Devesa, M y Sanz, } \\
\text { J.A. (2012) }\end{array}$ & $\begin{array}{l}\text { El impacto económico } \\
\text { de la celebración de } \\
\text { la Semana Santa en } \\
\text { Palencia }\end{array}$ & $\begin{array}{l}\text { Estimar impacto } \\
\text { económico de la Semana } \\
\text { Santa en Palencia }\end{array}$ & $\begin{array}{l}\text { Estudio de impacto } \\
\text { Económico a través del } \\
\text { método de los efectos. } \\
\text { Utilización de las TIO para } \\
\text { Castilla y León. } 2007 .\end{array}$ & $2.413 .334,96 €$ \\
\hline $\begin{array}{l}\text { Palma, Palma y } \\
\text { Aguado (2013) }\end{array}$ & $\begin{array}{l}\text { Determinants of } \\
\text { cultural and popular } \\
\text { celebration } \\
\text { attendance: the case } \\
\text { study of Sevilla Spring } \\
\text { Fiestas }\end{array}$ & $\begin{array}{l}\text { Análisis de asistencia a la } \\
\text { celebración de las Fiestas } \\
\text { de Primavera en Sevilla. } \\
\text { FPS como bien cultural }\end{array}$ & $\begin{array}{l}\text { Estudio de demanda basado } \\
\text { en un modelo de datos de } \\
\text { recuento. } \\
\text { Encuestas a asistentes } \\
(594)\end{array}$ & $\begin{array}{l}\text { Asistencia relacionada } \\
\text { con lazos } \\
\text { institucionales y nivel } \\
\text { de conocimientos }\end{array}$ \\
\hline $\begin{array}{l}\text { Grupo Satélite } \\
(2013)\end{array}$ & $\begin{array}{l}\text { El valor económico de } \\
\text { la Semana Santa en } \\
\text { la Antigua Guatemala }\end{array}$ & $\begin{array}{l}\text { Estimar impacto } \\
\text { económico de la } \\
\text { celebración de la Semana } \\
\text { Santa. }\end{array}$ & $\begin{array}{l}\text { Realización de } 1500 \\
\text { encuestas en la S.S. } 2011 \text { a } \\
\text { residentes y visitantes y } \\
\text { aplicación del método de los } \\
\text { efectos para calcular el } \\
\text { impacto económico }\end{array}$ & $\begin{array}{l}\text { Efecto total } \\
672,7 \text { millones de } \\
\text { quetzales } \\
\text { guatemaltecos* }\end{array}$ \\
\hline
\end{tabular}

* aproximadamente 61,3 millones de euros al tipo de cambio de 23 de octubre de 2013

Fuente: Elaboración propia a partir de los trabajos citados.

\subsection{El impacto económico de las Fiestas de Primavera de Sevilla}

No tratamos con este apartado llevar a cabo un análisis profundo del impacto económico de las Fiestas de Primavera de Sevilla (FPS) sobre la propia ciudad y su entorno, entendido éste en un sentido amplio. Tampoco se trata de plantear 
con amplitud el marco metodológico completo para la realización de los estudios de impacto aplicado a eventos de esta naturaleza ${ }^{7}$.

Naturalmente, ese marco debe quedar esbozado al objeto de explicar los elementos consustanciales de un estudio de impacto tipo. Pero, por razones de espacio, queremos dedicar mayor atención a los resultados del estudio que llevamos a cabo ${ }^{8}$ y sobre todo a realizar una valoración de los mismos, al objeto de puntualizar los que consideramos resultan más relevantes y matizar interesadas interpretaciones.

\subsubsection{Un apunte metodológico}

Los estudios de impacto suponen un modo de aproximar la rentabilidad de determinados eventos en el corto plazo. La aproximación se circunscribe al valor económico, dejando al margen la estimación del valor cultural.

Seaman (2003) plantea una metodología de cálculo a partir de la suma de tres efectos. En primer lugar, los efectos directos, que se derivan del volumen total de gasto realizado, tanto por instituciones públicas como privadas, para la celebración del evento. Hablamos, fundamentalmente del "arreglo del escenario" y la preparación de los elementos esenciales de las fiestas. En segundo lugar, los efectos indirectos, producto del gasto efectuado por los participantes en la Semana Santa y en la Feria de Abril, ya sean naturales de Sevilla o personas que visiten la ciudad con motivo de las Fiestas.

Por último, se estiman los efectos inducidos para la economía en su conjunto. Es esencial para el cálculo de esta tercera categoría de efectos contar con multiplicadores sectoriales, calculados a partir de una tabla input-output. A continuación vamos a detallar algo más estos efectos para el caso de las Fiestas de Primavera de Sevilla.

\subsubsection{Efectos directos}

Incorporamos en los efectos directos todos los gastos necesarios para la celebración del evento, procedentes de instituciones públicas y privadas. Con relación al gasto procedente de Instituciones públicas, éste se deriva esencialmente del realizado por el Ayuntamiento de la ciudad, tanto en el caso de la Semana Santa como en la Feria de Abril. Los datos para el estudio proceden de la Delegación de Fiestas Mayores del Ayuntamiento de Sevilla.

El gasto privado incorporado a los efectos directos procede de instituciones o agentes económicos distintos según nos refiramos a la Semana Santa o a la Feria de Abril. En el primer caso, procede del Consejo General del Hermandades y

\footnotetext{
${ }^{7}$ Puede verse este análisis metodológico en extenso en Palma, García y Palma, 2008.

${ }^{8}$ Ver Palma Martos, L. (dir) (2010a) y (2010b).
} 
Cofradías, institución que juega un papel esencial de coordinación y organización del evento, y de las propias Hermandades, en un número de cincuenta y nueve. Los datos para el estudio se derivan del presupuesto global del Consejo de Hermandades y Cofradías, facilitado por esta institución y del presupuesto de las Hermandades, estimado éste a partir de una muestra realizada "ad hoc", cuidándonos de que estuvieran representadas las diversas tipologías existentes.

En el caso de la Feria de Abril, y dada la naturaleza del evento, los agentes involucrados son más diversos. Así, tenemos los propietarios y socios de las "casetas"; los propietarios de carruajes y caballos y los gastos derivados de la múltiple variedad de negocios que se instalan en la Feria (bares, venta ambulante de naturaleza diversa, parque de atracciones). Los datos, en el caso de las "casetas" proceden de una encuesta "ad hoc", que recoge la diversa tipología de las mismas (tamaño, filiación). El gasto realizado por los propietarios de carruajes y caballos y de negocios instalados en "el Real de la Feria" fue proporcionado desde la Delegación de Fiestas Mayores del Ayuntamiento. En la Tabla A1 del anexo se ofrecen pormenorizados el total de gastos directos, según tipo de gasto, para los acontecimientos que conforman las FPS. En la Tabla 6 se recogen como uno de los componentes del Efecto Local Total.

\subsubsection{Efectos indirectos}

En esta fase se trata de estimar el gasto llevado a cabo por todos los participantes en las FPS. Esto supone conocer, por un lado, el número de participantes, y por otro, el gasto medio de éstos. A partir de estos datos podremos estimar el gasto total. En este punto del estudio surgen algunas dificultades metodológicas. Hay que ser cautelosos a la hora de incluir determinados gastos que podrían suscitar problemas de doble contabilización (por ejemplo, el pago de alquiler de sillas o "palcos" para ver las procesiones de Semana Santa se integra en el Presupuesto del Consejo General de Hermandades, ya considerado como gasto directo).

Tabla 3

Número estimado de visitantes diarios

\begin{tabular}{|l|c|c|}
\hline \multicolumn{1}{|c|}{ TIPO } & SEMANA SANTA & FERIA DE ABRIL \\
\hline Locales & 349.748 & 358.014 \\
\hline Visitantes de la provincia de Sevilla & 32.625 & 72.000 \\
\hline No residentes que pernoctan en hoteles & 21.803 & 19.502 \\
\hline $\begin{array}{l}\text { No residentes que pernoctan en sus domicilios o en } \\
\text { casas de familiares y/o amigos }\end{array}$ & 21.286 & 43.408 \\
\hline TOTAL VISITANTES DIARIOS & $\mathbf{4 2 5 . 4 6 2}$ & $\mathbf{4 9 2 . 9 2 4}$ \\
\hline
\end{tabular}

Fuente: Elaboración propia a partir de los resultados de nuestra encuesta y Ayuntamiento de Sevilla (2009). Informe socioeconómico y anuario estadístico de la Ciudad de Sevilla 2006-2008. 
Tenemos también abierta la cuestión de si incorporar o no como fuente de gasto a los participantes locales. Hemos considerado la naturaleza de la participación de los locales y su grado de implicación en los eventos y hemos concluido en incorporar su gasto a los efectos indirectos. Entendemos que el gasto de los locales se ve incrementado de forma específica por la celebración de las FPS, es decir, está directamente relacionado con la esencia de los eventos, no siendo sustituible por otro tipo de gasto.

La estimación del número de visitas (ver Tabla 3) se ha realizado a partir de datos de visitantes y pernoctaciones proporcionados por la Consejería de Turismo y Deporte de la Junta de Andalucía (2009) y el propio Ayuntamiento de Sevilla (2009). El gasto medio por participante se ha obtenido mediante una encuesta llevada a cabo los días de celebración de los eventos. Se completaron un total de 594 cuestionarios (310 correspondientes a la Semana Santa y 284 a la Feria de Abril; de ellos 314 procedían de participantes locales y 280 de no locales $^{9}$ (ver Tabla 4). El efecto indirecto se corresponde con el gasto realizado por los visitantes. A partir de las Tablas 3 y 4 obtenemos ese gasto. Hemos estimado, por un lado, Tabla 3, el número de visitantes, según las diversas tipologías consideradas; y por otro, Tabla 4, el gasto medio por persona para las mismas tipologías referidas en la Tabla 3. El Efecto Indirecto se obtiene multiplicando, para cada tipología, el número estimado de visitantes por el gasto medio; y esto tanto para la Semana Santa como para la Feria de Abril. El Efecto indirecto para cada evento se incorpora a la Tabla 6 como segundo componente del Efecto Local Total.

Tabla 4

Gasto medio estimado

\begin{tabular}{|l|c|c|}
\hline \multicolumn{2}{|c|}{ TIPO DE PARTICIPANTE } & \multicolumn{2}{c|}{ EVENTO } \\
\hline NO RESIDENTES QUE SE ALOJAN EN HOTELES & SEMANA SANTA & FERIA DE ABRIL \\
\hline Gasto medio total s/cuestionario (A) & $478,77 €$ & $719,36 €$ \\
\hline Número de personas en el grupo (B) & 2,05 & 2,43 \\
\hline Número de dias de asistencia (C ) & 3,52 & 2,19 \\
\hline Gasto medio por persona (D = A/B) & $233,55 €$ & $296,03 €$ \\
\hline Gasto medio por persona y día (D/C) & $66,35 €$ & $135,17 €$ \\
\hline NO RESIDENTES QUE SE ALOJAN EN DOMICILIO O & SEMANA SANTA & FERIA DE ABRIL \\
\hline CASAS DE FAMILIARES Y/O AMIGOS & $126,78 €$ & $345,51 €$ \\
\hline Gasto medio total s/cuestionario (A) & 2,05 & 2,57 \\
\hline Número de personas en el grupo (B) & 2,61 & 2,52 \\
\hline Número de días de asistencia (C ) & $61,84 €$ & $134,44 €$ \\
\hline Gasto medio por persona (D = A/B) & $23,69 €$ & $53,35 €$ \\
\hline Gasto medio por persona y día (D/C) & & \\
\hline
\end{tabular}

${ }^{9}$ La muestra fue obtenida siguiendo el procedimiento de Levy y Lameshow (1991) con un error muestral máximo del $5 \%$ y un nivel de confianza del $95 \%$. 
Tabla 4 (continuación)

Gasto medio estimado

\begin{tabular}{|l|c|c|}
\hline \multicolumn{1}{|c|}{ TIPO DE PARTICIPANTE } & \multicolumn{2}{c|}{ EVENTO } \\
\hline VISITANTES DE LA PROVINCIA DE SEVILLA & SEMANA SANTA & FERIA DE ABRIL \\
\hline Coste total medio s/cuestionario (A) & 117,92 & $345,51 €$ \\
\hline Número de personas en el grupo (B) & 2,05 & 2,57 \\
\hline Número de días de asistencia (C ) & 2,61 & 2,52 \\
\hline Gasto total medio por persona (D = A/B) & $57,52 €$ & $134,44 €$ \\
\hline Gasto medio por persona y día (D/C) & $22,04 €$ & $53,35 €$ \\
\hline PARTICIPANTES LOCALES & SEMANA SANTA & FERIA DE ABRIL \\
\hline Coste total medio s/cuestionario (A) & $145,01 €$ & $477,66 €$ \\
\hline Número de personas en el grupo (B) & 2,24 & 3,63 \\
\hline Número de dias de asistencia (C ) & 4,03 & 3,4 \\
\hline Gasto total medio por persona (D = A/B) & $66,74 €$ & $131,58 €$ \\
\hline Gasto medio por persona y día (D/C) & $16,56 €$ & $38,7 €$ \\
\hline
\end{tabular}

Nota: La estimación del gasto medio, el número de personas en cada grupo y los días de asistencia se toman del cuestionario.

Fuente: Elaboración propia.

\subsubsection{Efectos inducidos}

A partir del cálculo de los efectos directos e indirectos, debemos estimar la difusión de esos gastos en el conjunto de sectores productivos, a fin de realizar la estimación del efecto total o impacto sobre el resto de la economía. Este efecto va a depender de la propensión de los locales a comprar productos locales o importados y del área geográfica considerada para el estudio.

Tabla 5

Multiplicadores estimados para las Fiestas de Primavera de la ciudad de Sevilla

\begin{tabular}{|l|l|l|}
\hline \multicolumn{1}{|c|}{ TIPO DE GASTO } & Interior & Total \\
\hline Gastos en infraestructura y mantenimiento & 2,099212 & 3,002599 \\
\hline Exorno & 1,877240 & 2,751751 \\
\hline Restauración y mantenimiento del patrimonio & 1,500328 & 3,179071 \\
\hline Transporte & 1,542830 & 2,411181 \\
\hline Confección de túnicas y complementos & 1,460465 & 3,032562 \\
\hline Actividades de limpieza & 1,260802 & 1,581354 \\
\hline Otros servicios a las empresas & 1,481512 & 1,986738 \\
\hline $\begin{array}{l}\text { Otras actividades recreativas, culturales y } \\
\text { deportivas (música) }\end{array}$ & 1,508291 & 1,833260 \\
\hline Actividades de saneamiento público & 1,386008 & 1,671733 \\
\hline Actividades sanitarias no de mercado & 1,186198 & 1,569481 \\
\hline Comida y bebida & 1,507749 & 2,253620 \\
\hline Hostelería y alojamientos & 1,406385 & 1,792275 \\
\hline Otros gastos corrientes & 1,467724 & 1,922535 \\
\hline Gastos de personal & 1,171693 & 1,682385 \\
\hline
\end{tabular}

Fuente: Elaboración propia. 
El marco de análisis input-output resulta adecuado para llevar a cabo la estimación señalada. En nuestro caso hemos utilizado los multiplicadores a partir de la Tabla Input-Output de Andalucía (2005). En la Tabla 5 quedan reflejados los multiplicadores estimados. Éstos nos permiten, según su naturaleza, calcular los efectos interiores y exteriores.

En las Tablas A2 y A3 del anexo se ofrece pormenorizadamente la estimación de los efectos inducidos, tanto interiores como exteriores. Se han obtenido al multiplicar, para cada una de las tipologías de gasto, el Efecto Local Total por el multiplicador correspondiente procedente de la Tabla 5. En la Tabla 6 se recogen los efectos inducidos totales a efectos de estimación global del impacto.

\subsubsection{Resultados del estudio}

Los resultados de la estimación se presentan en la Tabla 6, en la que se ofrece una visión global del impacto económico de las FPS.

Tabla 6

Impacto económico estimado de las Fiestas de Primavera de Sevilla (en Euros)

\begin{tabular}{|l|r|r|r|}
\hline & SEMANA SANTA & FERIA DE ABRIL & \multicolumn{1}{c|}{ TOTAL FPS } \\
\hline EFECTO LOCAL TOTAL & $\mathbf{7 6 . 5 0 8 . 9 5 6} €$ & $\mathbf{1 9 5 . 2 7 2 . 0 2 6} €$ & $\mathbf{2 7 1 . 7 8 0 . 9 8 2} €$ \\
\hline Efecto directo & $8.380 .858 €$ & $36.734 .422 €$ & $45.115 .280 €$ \\
\hline Efecto indirecto & $68.128 .098 €$ & $158.537 .604 €$ & $226.665 .702 €$ \\
\hline EFECTO INDUCIDO TOTAL & $\mathbf{1 6 3 . 7 9 8 . 0 8 8} €$ & $\mathbf{4 8 0 . 2 4 4 . 4 1 1} €$ & $\mathbf{6 4 4 . 0 4 2 . 4 9 9} €$ \\
\hline Efecto inducido interior & $115.333 .940 €$ & $294.960 .430 €$ & $410.294 .370 €$ \\
\hline Efecto inducido exterior & $48.462 .148 €$ & $185.283 .981 €$ & $233.746 .129 €$ \\
\hline IMPACTO ECONÓMICO TOTAL & $\mathbf{2 4 0 . 3 0 5 . 0 4 4} €$ & $\mathbf{6 7 5 . 5 1 6 . 4 3 7} €$ & $\mathbf{9 1 5 . 8 2 1 . 4 8 1} €$ \\
\hline
\end{tabular}

Fuente: Elaboración propia.

Así, tenemos que el efecto local total (directo más indirecto) de la Semana Santa, 76,5 millones de euros, genera un efecto inducido de 163,8 de los cuales aproximadamente el $70 \%$ afectan a la economía andaluza. En el caso de la Feria de Abril, los 195,2 millones de euros de efecto local generan un efecto inducido de 480 millones; aproximadamente el $60 \%$ de ese efecto recae sobre la economía andaluza. El impacto económico se cifra en 915.8 millones de euros.

Nos interesa calibrar la dimensión del impacto económico en el contexto de la economía de la ciudad de Sevilla y de su provincia. Para ello tomamos como referencia los datos del PIB de Sevilla (13\% del PIB de Andalucía, 19.760 millones de euros) y de la Provincia (34,32 millones de euros) (IEA, 2008). A partir de estos datos y de los datos de la Tabla 6 construimos la Tabla 7. Como 
vemos, el tamaño relativo del impacto económico en porcentaje del PIB para la ciudad es de un $4,66 \%$ y para la provincia de un $2,69 \%$.

Tabla 7

Tamaño relativo del impacto económico (porcentaje del PIB)

\begin{tabular}{|l|c|c|c|}
\hline & SEMANA SANTA & FERIA DE ABRIL & TOTAL FPS \\
\hline Comparado con el PIB de la ciudad & $1,2 \%$ & $3,46 \%$ & $4,66 \%$ \\
\hline Comparado con el PIB de la provincia & $0,7 \%$ & $1,99 \%$ & $2,69 \%$ \\
\hline
\end{tabular}

Fuente: Elaboración propia.

Se observa que la Feria de Abril supera ampliamente a la Semana Santa como generadora de impacto económico, tanto a nivel local como provincial. Otra perspectiva de análisis nos la proporciona el uso de los multiplicadores medios estimados (ver Tabla 8).

Tabla 8

Multiplicadores medios estimados

\begin{tabular}{|l|c|c|c|}
\hline & SEMANA SANTA & FERIA DE ABRIL & TOTAL FPS \\
\hline Interior & 1,48 & 1,51 & 1,50 \\
\hline Exterior & 0,66 & 0,95 & 0,87 \\
\hline Total & 2,14 & 2,46 & 2,37 \\
\hline
\end{tabular}

Fuente: Elaboración propia.

Vemos que la Semana Santa tiene un efecto multiplicador total de 2,14 (interior, 1,48). En el caso de la Feria de Abril el multiplicador total es 2,46 y el interior 1,51. Queremos indicar con esta perspectiva que cada euro gastado en la celebración de la Semana Santa en la ciudad de Sevilla genera 2,14 euros adicionales, de los cuales 1,48 lo hacen en el marco de la economía andaluza. Y cada euro gastado en la celebración de la Feria de Abril genera 2,46 euros adicionales, permaneciendo 1,51 en el marco de la economía andaluza. Desde esta perspectiva, ambos eventos tienen una incidencia similar sobre la Economía.

\subsubsection{Valoración de resultados}

La estimación del impacto económico de las FPS nos permite formular algunas valoraciones sobre estas celebraciones. En primer lugar, la mayor dimensión del efecto local de la feria de Abril (2,6 veces más) que el de la Semana Santa. En segundo lugar, aún mayor es la diferencia de los efectos inducidos. El de la feria de Abril triplica el de la Semana Santa. Se explican estas diferencias por el 
mayor nivel de los multiplicadores de la Feria de Abril (tanto el interior como el exterior).

Podríamos también vincular la diferencia de los impactos a la propia naturaleza de los eventos. Así, la Semana Santa podría entenderse como un bien público, esencialmente, mientras que resulta evidente la naturaleza de bien eminentemente privado de la feria de Abril. El tipo de estudio realizado, un estudio de impacto, conlleva la dificultad de captar el valor de los bienes y servicios de no mercado, consustancial en mayor grado a la Semana Santa.

\section{CONCLUSIONES}

El principal objetivo de este artículo es determinar la relación entre cultura y economía a través del análisis de las Fiestas de Primavera de Sevilla, prototipo de bien cultural. Las FPS como bien cultural entraña una gran complejidad por ser de naturaleza compuesta, al aunar características de los distintos ámbitos de la economía de la cultura; asimismo, destaca por poseer un alto valor de patrimonio inmaterial.

La literatura empírica que explora este tipo de bienes ha crecido en los últimos años centrándose fundamentalmente en estudios de impacto económico sobre un evento de carácter único (opera, cine, festivales). En contraposición, este artículo supone una contribución original en la medida que se aborda un estudio de impacto económico sobre un evento complejo de naturaleza compuesta, enfoque ausente hasta la fecha a excepción del trabajo de Palma et al. (2013), como ejemplo de revalorización económica a través de la cultura e integración entre ambas áreas.

Las Fiestas de Primavera constituyen el principal evento cultural de la ciudad cada año y su principal capital cultural. Las FPS son expresiones significativas de la vida social, económica, política y artística de la ciudad lo que implica un alto grado de participación y articulación social. Representan un fuerte poder de atracción turística tanto para los habitantes del área metropolitana y provincia, como para el resto de España y a nivel internacional. Desde esta perspectiva son especialmente interesantes desde el punto de vista del turismo cultural.

La celebración de las FPS contribuye a poner en valor y reforzar tanto una parte importante del patrimonio histórico material e inmaterial de Sevilla, por tanto, el capital cultural, como el capital social de la ciudad, nacido de las interrelaciones entre los diversos actores económicos, no económicos e institucionales, que hacen posible no sólo una valoración en términos económicos sino socio-culturales, emocionales y de identidad.

Desde el punto de vista de su contribución económica, las FPS tienen un impacto total estimado en el año 2009 de 915.8 millones de Euros que representa un 4,66\% del PIB de Sevilla y un 2,69\% del PIB provincial. La celebración de 
las FPS tiene un efecto multiplicador medio estimado de 2,37, siendo el efecto multiplicador regional de 1,5 .

La Feria de Abril supera ampliamente a la Semana Santa como generadora de impacto económico tanto a nivel local como regional en una relación de 3 a 1. Esta diferencia puede explicarse por la naturaleza de los eventos, más privada en el caso de la Feria de Abril frente a un mayor componente de bien público de la Semana Santa. Este tipo de estudios conlleva la dificultad de captar el valor de los bienes y servicios de no mercado, más presentes en la Semana Santa.

El resultado de esta investigación revela que el impacto económico no es sólo el efecto de la llegada de turistas y visitantes, con el consiguiente gasto asociado a los mismos, sino que tanto los residentes como las instituciones locales, de carácter público y privado, desempeñan un papel clave en la generación de flujos económicos.

Por tanto hay que admitir, que eventos culturales como las FPS son algo más que simples expresiones de la idiosincrasia, creatividad, y valores culturales de una ciudad, en este caso de Sevilla. También generan un importante volumen de actividad económica cuyo impacto va más allá de los límites de la actividad local, y tienen unos importantes efectos difusores sobre la economía regional y en menor medida en la economía más allá de la Comunidad Autónoma. Esto explica el énfasis de las autoridades locales en potenciar la celebración de este tipo de eventos como fórmula de regeneración urbana y potenciadora de la economía local, fundamentalmente interesantes en un contexto de contracción económica como el actual.

\section{REFERENCIAS BIBLIOGRÁFICAS}

ANALISTAS ECONÓMICOS DE ANDALUCÍA. (2009). Semana Santa. Efectos socioeconómicos de la Semana Santa en la ciudad de Córdoba. Agrupación de Hermandades y Cofradías de Córdoba. Ayuntamiento de Córdoba Unicaja Córdoba. http://www.hermandadesdecordoba.es/descargas/Efectos\%20socioeconomicos\%20 de\%20la\%20Semana\%20Santa.pdf. [Último acceso: octubre 2013].

AYUNTAMIENTO DE SEVILLA (2009) Informe socieconómico y anuario estadístico de la ciudad de Sevilla 2006-2008. Sevilla: Ayuntamiento de Sevilla. $\quad$ http://www.sevilla.org/ayuntamiento/areas/area-de-hacienda-yadministracionpublica/ servicio-estadistica/archivos/anuarios/informe-2008 [Último acceso: septiembre 2013]

BUCHANAN, J. (1965)."An economic theory of clubs"en Económica, 32, pp.1-14.

CHEW, M.S.Y. (1998). "Culture, circles and commercials: Publicity, common knowledge and social coordination" en Rationality and Society, 10, pp.47-75. 
CONFEDERACIÓN DE EMPRESARIOS DE SEVILLA Y CÁMARA DE COMERCIO DE SEVILLA (2008). El impacto de la celebración de la Semana Santa sobre la ciudad de Sevilla. Sevilla 2008. Sevilla: Cámara de Comercio http://www.fundacioncamaradesevilla.es/documentos/516879ef0440ebcb38eb 3dd624b66288.pdf. [Último acceso: octubre 2013].

CONSEJERÍA DE TURISMO Y DEPORTE DE LA JUNTA DE ANDALUCÍA (2009). Informe mensual de coyuntura turística de Andalucía. http://www.juntadeandalucia.es/turismoycomercio/opencms/estadisticas/e stadisticas-oficiales-de-la-consejeria-de-turismo-comercio-ydeporte/index.html\#balance [ Último acceso: septiembre 2013]

DEL BARRIO, M.J.; DEVESA, M.; HERRERO, L.C. (2012). Evaluating intangible cultural heritage: the case of cultural festivals en City, Culture and Society, 3, pp.235-244.

GRUPO SATÉLITE (2013) El valor económico de la Semana Santa en La Antigua Guatemala. Guatemala: Ed. Cultura.

GURSOY, D.; KIM, K. y UYSAL, M. (2004). "Perceived impacts and special events by organizers: an extension and validation" en Tourism Management, 25, pp.171-181.

INSTITUTO DE ESTADÍSTICA Y CARTOGRAFÍA DE ANDALUCÍA (2008a). Tablas input-output de Andalucía 2005. Sevilla: Junta de Andalucía, Consejería de Economía, Innovación, Ciencia y Empleo. http://www.juntadeandalucia.es/ institutodeestadisticaycartografia/mioan/mioan2005/index.htm [Último acceso: septiembre 2013]

INSTITUTO DE ESTADÍSTICA Y CARTOGRAFÍA DE ANDALUCÍA (2008b). Contabilidad regional anual de Andalucía. http://www.juntadeandalucia.es/ institutodeestadisticaycartografia/craa/index.ht [Último acceso: septiembre 2013]

LAFUENTE, V.; DEVESA, M. y SANZ, J.A. (2012) "El impacto económico de la celebración de la Semana Santa en Palencia”. IV Workshop en Economía y Gestión de la Cultura. Bilbao, 29 y 30 de noviembre de 2012.

LAZZERETTI, L. (2008). The cultural districtualization model. En Cooke, P. y Lazzeretti, L. (eds): Creative cities, cultural clusters and local development (pp. 93-120). Cheltenham: Edward Elgar.

LAZZERETTI, L. (2011). "Culture as a source for growth and change: some evidences from cultural clusters in Andalusia". En Asheim, B.; Boschma, R.; Cooke, P.; Martín, R.; Schwartz, D. y Todtling, F. (eds): Handbook of regional innovation and growth (pp.350-362). Cheltenham: Edward Elgar

LEVY, P. y LAMESHOW, S. (1991). Sampling of populations: Methods and applications. Wiley Interscience.

MORENO, I. (1999). Las hermandades andaluzas: una aproximación desde la Antropología. Sevilla: Universidad de Sevilla.

MORENO, I. (2001). La Semana Santa de Sevilla. Conformación, mixtificación y significaciones. Sevilla: Biblioteca de temas sevillanos.

NEA (2010). Life from your neighborhood. A national study of outdoor festivals. Research report $n^{\circ}$. 51. Washington: National Endowment for the Arts (NEA).

O'HAGAN, J. (1996). "Access to and participation in the arts: The case of those with low income/educational attainment"en Journal of Cultural Economics, 20 (4), pp.260-282.

PALMA, L; GARCÍA A. y PALMA, M .L. (2008): “Una propuesta metodológica para la evaluación del impacto económico de las Fiestas de Primavera en la ciudad de Sevilla en Cuadernos de Economía de la Cultura, 9, año VI, pp. 7-39. 
PALMA, L.; AGUADO, L. F.; GARCÍA, A.; MARTíN, J. L.; PALMA, M. L.; RIDAO, M. L. y VILLAR, C. (2010 a). Perfil de los asistentes e impacto económico de la Semana Santa de Sevilla en el año 2009: Informe de Investigación. Delegación de Fiestas Mayores. Ayuntamiento de Sevilla

PALMA, L.; AGUADO, L. F.; GARCÍA, A.; MARTíN, J. L.; PALMA, M. L.; RIDAO, M. L. y VILLAR, C. (2010 b). Perfil de los asistentes e impacto económico de la Feria de Abril de Sevilla en el año 2009: Informe de Investigación. Delegación de Fiestas Mayores. Ayuntamiento de Sevilla

PALMA.M. L.; PALMA, L. y AGUADO, L.F. (2013) "Determinants of cultural and popular celebration attendance: The case study of Sevilla Spring Fiestas" en Journal of Cultural Economics, 37 (1), pp 87-107.

SEAMAN, B. (2003). The economic impact of the arts. En Towse, R. (ed), A handbook of Cultural Economics (pp. 224-231). Cheltenham: Edward Elgar.

THROSBY, D. (1999)." Cultural capital” en Journal of Cultural Economics, 23 (1), pp.312.

UNESCO (2009). Marco de estadísticas culturales de la Unesco 2009, Montreal (Canadá): Instituto de Estadística de la UNESCO. http://www.uis.unesco.org/culture/ Documents/framework-cultural-statistics-culture-2009-en.pdf [Último acceso: septiembre 2013]. 


\section{ANEXO}

Tabla A1

Efecto directo

(En euros)

\begin{tabular}{|c|c|c|c|}
\hline Tipo de gasto & Semana Santa & Feria de Abril & Total de Efecto Directo \\
\hline Seguros & & 430.135 & 430.135 \\
\hline Seguridad & & 2.282 .892 & 2.282 .892 \\
\hline Otros medios de Transporte & & 5.600 .000 & 5.600 .000 \\
\hline Administración Pública & & 4.862 .227 & 4.862 .227 \\
\hline Publicidad & & 204.455 & 204.455 \\
\hline $\begin{array}{l}\text { Gastos en Infraestructura y } \\
\text { Mantenimiento }\end{array}$ & 1.062 .360 & 14.057.976 & $15,120.336$ \\
\hline Ornamentación decoración municipal & 855.496 & 2.847 .745 & 3.703 .241 \\
\hline Restauración y Conservación & 574.451 & & 574.451 \\
\hline Transporte & 1.692 .340 & 478.880 & 2.171 .220 \\
\hline Actividades de limpieza & 877.028 & 1.052 .433 & 1.929 .461 \\
\hline Otros servicios a las empresas & 235.938 & & 235.938 \\
\hline $\begin{array}{l}\text { Otras actividades recreativas, culturales } \\
\text { y deportivas (música) }\end{array}$ & 648.488 & 3.156 .437 & 3.804 .925 \\
\hline Actividades de saneamiento público & 76.608 & 138.690 & 215.298 \\
\hline Actividades sanitarias no de mercado & 6.552 & 5.220 & 11.772 \\
\hline Otros gastos corrientes & 665.988, & & 665.988 \\
\hline Gastos de personal & 1.685 .609 & 1.617 .332 & 3.302 .941 \\
\hline TOTAL & 8.380 .858 & 36.734 .422 & 45.115 .280 \\
\hline
\end{tabular}

Fuente: Elaboración propia a partir de datos de encuestas y Ayuntamiento (2009).

Tabla A2

Impacto económico estimado de la Semana Santa (en euros)

\begin{tabular}{|c|c|c|c|c|c|}
\hline \multirow{3}{*}{ TIPO DE GASTO } & \multicolumn{5}{|c|}{ TOTAL EFECTO LOCAL=EFECTO DIRECTO + EFECTO INDIRECTO } \\
\hline & \multirow{2}{*}{$\begin{array}{l}\text { TOTAL } \\
\text { EFECTO } \\
\text { LOCAL }\end{array}$} & \multicolumn{3}{|c|}{ EFECTO INDUCIDO } & \multirow{2}{*}{$\begin{array}{c}\text { TOTAL } \\
\text { IMPACTO } \\
\text { ECONÓMICO }\end{array}$} \\
\hline & & TOTAL & INTERIOR & EXTERIOR & \\
\hline $\begin{array}{l}\text { Confección de túnicas y } \\
\text { complementos }\end{array}$ & 12.881 .644 & 39.064 .383 & 18.813 .190 & 20.251 .193 & 51.946 .027 \\
\hline $\begin{array}{l}\text { Gastos de infraestructura y } \\
\text { mantenimiento }\end{array}$ & 1.062 .360 & 3.189 .841 & 2.230 .119 & 959.722 & 4.252 .201 \\
\hline Exorno & 855.496 & 2.354 .112 & 2.354 .112 & 0 & 3.209 .608 \\
\hline Restauración y conservación & 574.451 & 1.826 .507 & 1.826 .507 & 0 & 2.400 .958 \\
\hline Transporte & 4.806 .221 & 11.588 .670 & 7.415 .183 & 4.173 .487 & 16.394 .891 \\
\hline Actividades de limpieza & 877.028 & 1.386 .892 & 1.105 .759 & 281.133 & 2.263 .920 \\
\hline Otros servicios a las empresas & 235.938 & 468.747 & 349.545 & 119.202 & 704.685 \\
\hline $\begin{array}{l}\text { Otras actividades de recreo, culturales } \\
\text { y deportivas }\end{array}$ & 27.604 .542 & 50.606 .302 & 41.635 .682 & 8.970 .620 & 78.210 .844 \\
\hline Actividades de saneamiento público & 76.608 & 128.068 & 106.179 & 21.889 & 204.676 \\
\hline Actividades sanitarias no de mercado & 6.552 & 10.283 & 7.772 & 2.511 & 16.835 \\
\hline Comida y bebida & 6.234 .246 & 14.049 .621 & 9.399 .678 & 4.649 .943 & 20.283 .867 \\
\hline Hostelería alojamiento & 10.830 .251 & 19.410 .789 & 15.231 .503 & 4.179 .286 & 30.241 .040 \\
\hline Otros gastos corrientes & 8.778 .010 & 16.876 .030 & 12.883 .695 & 3.992 .335 & 25.654 .040 \\
\hline Costes de personal & 1.685 .609 & 2.835 .843 & 1.975 .016 & 860.827 & 4.521 .452 \\
\hline TOTAL & 76.508 .956 & 163.796 .088 & 115.333 .940 & 48.462.1498 & 240.305 .044 \\
\hline
\end{tabular}

Fuente: Elaboración propia a partir de datos de encuestas, Ayuntamiento (2009) e Instituto de Estadística y Cartografía de Andalucía (2008a). 


\section{Tabla A3}

Impacto económico estimado de la Feria de Abril

(en euros)

\begin{tabular}{|c|c|c|c|c|c|}
\hline \multirow{3}{*}{ TIPO DE GASTO } & \multicolumn{5}{|c|}{ TOTAL EFECTO LOCAL=EFECTO DIRECTO + EFECTO INDIRECTO } \\
\hline & \multirow{2}{*}{$\begin{array}{l}\text { TOTAL } \\
\text { EFECTO } \\
\text { LOCAL }\end{array}$} & \multicolumn{3}{|c|}{ EFECTO INDUCIDO } & \multirow{2}{*}{$\begin{array}{c}\text { TOTAL } \\
\text { IMPACTO } \\
\text { ECONÓMICO }\end{array}$} \\
\hline & & TOTAL & INTERIOR & EXTERIOR & \\
\hline $\begin{array}{l}\text { Gastos de infraestructura y } \\
\text { mantenimiento }\end{array}$ & 14.057.976 & 42.210 .465 & 29.510 .672 & 12.699 .793 & 56.268 .441 \\
\hline Publicidad y relaciones $p$ & 204.455 & 562.609 & 383.811 & 178.798 & 767.064 \\
\hline Decoración & 2.847 .745 & 7.447 .806 & 4.300 .624 & 3.147 .182 & 10.295 .551 \\
\hline Transporte & 19.180 .543 & 46.247 .762 & 29.592 .318 & 16.655 .444 & 65.428 .305 \\
\hline Actividades de Limpieza & 1.052 .433 & 1.664 .270 & 1.326 .910 & 337.360 & 2.716 .703 \\
\hline Seguros & 430.135 & 839.300 & 725.905 & 113.395 & 1.269 .435 \\
\hline $\begin{array}{l}\text { Otras actividades de recreo, culturales } \\
\text { y deportivas }\end{array}$ & $41.190 .968 €$ & 75.513 .754 & 62.127 .966 & 13.385 .788 & 116.704 .722 \\
\hline Actividades de saneamiento público & 138.690 & 231.852 & 192.225 & 39.627 & 370.542 \\
\hline $\begin{array}{l}\text { Actividades sanitarias no de mercado } \\
\text { y veterinarias }\end{array}$ & 5.220 & 8.193 & 6.192 & 2.001 & 13.413 \\
\hline Seguridad y vigilancia & 2.282 .892 & 3.434 .811 & 2.658 .376 & 776.435 & 5.717 .703 \\
\hline Gastos de personal & 1.617 .332 & 2.720 .976 & 1.895 .017 & 825.959 & 4.338 .308 \\
\hline Administración Pública & 4.862 .227 & 7.416 .612 & 6.155 .934 & 1.260 .678 & 12.278 .839 \\
\hline Otros tipos de transporte & 5.600 .000 & 13.266 .618 & 8.716 .898 & 4.549 .720 & 18.866 .618 \\
\hline Indumentaria y accesorios & 77.581 .446 & 235.270 .546 & 113.304 .987 & 121.965 .559 & 312.851 .992 \\
\hline Alojamientos y Hoteles & 24.219 .964 & 43.408 .837 & 34.062 .595 & 9.346 .242 & 67.628 .801 \\
\hline TOTAL & 195.272.026 & 480.244 .411 & 294.960 .430 & 185.283.981 & 675.516 .437 \\
\hline
\end{tabular}

Fuente: Elaboración propia a partir de datos de encuestas, Ayuntamiento (2009) e Instituto de Estadística y Cartografía de Andalucía (2008a). 